\title{
Transport fehlgefalteter Proteine von der Peripherie in das Gehirn
}

Fragestellung: Es werden acht Autopsiefälle von Patienten beschrieben, die etwa 30 Jahre nach dem Erhalt von Wachstumshormonpräparaten aus der Hypophyse von menschlichen Kadavern an der iatrogenen Form der Creutzfeldt-Jakob Krankheit (iCJD) verstorben sind und von denen fast alle auch überraschend Anzeichen für Amyloid- $\beta$-Pathologie aufweisen.

Hintergrund: Das Prionprotein ist ein körpereigenes Protein, das sich fehlfalten und zu einer tödlichen Neurodegeneration führen kann. Prionerkrankungen wie die Creutzfeldt-Jakob Krankheit (CJD) können sporadischen, familiären oder infektiösen Ursprungs sein. Dazu zählt auch die iatrogene Übertragung durch die Behandlung mit prionkontaminiertem Wachstumshormon, das von 1958 bis 1985 noch aus menschlichen Kadavern gewonnen wurde. Es besteht ein Interesse bezüglich der potenziellen „prionähnlichen“ Übertragbarkeit anderer neurodegenerativer Krankheiten, wie beispielsweise Morbus Alzheimer, bei denen sich ebenfalls körpereigene Proteine fehlfalten und aggregieren.

Jaunmuktane Z, Mead S, Ellis $M$ et al. Evidence for human transmission of amyloid- $\beta$ pathology and cerebral amyloid angiopathy. Nature 2015; 525: $247-50$
Patienten und Methodik: Acht Autopsiefälle von iCJD, die bei einer Behandlung im Kindesalter mit humanem Wachstumshormon aus Kadavern versehentlich mit Prionen infiziert worden waren und im Alter zwischen 36 und 51 verstorben sind, wurden mittels Histologie und Immunoblot auf Amyloid- $\beta$-Pathologie hin untersucht. Die pathologischen Befunde wurden mit denen aus einer Kohorte von 116 CJD-Fällen verglichen, die an der sporadischen, varianten oder erblichen Form von CJD verstorben sind.

Ergebnisse: Sechs der acht iCJD-Fälle wiesen eine Amyloid- $\beta$ Pathologie auf, die zum Teil Amyloid- $\beta$-Plaques und zerebrale Amyloid-Angiopathie (CAA) beinhaltete und für diese Altersgruppe nicht bei der Vergleichskohorte auftrat. Keiner der Patienten trug das APOE $\varepsilon 4$ - oder TREM2 R47H-Allel oder wies Mutationen in 16 anderen Genen auf, die eine early-onset Alzheimer-Erkrankung, CAA oder andere neurodegenerative Erkrankungen verursachen.

Schlussfolgerungen: Die Beobachtungen lassen darauf schließen, dass es Mechanismen gibt, die den Transport von Prionen, A $\beta$-Aggregaten und vermutlich auch von anderen fehlgefalteten Proteinen wie Tau aus der Peripherie in das Gehirn ermöglichen. Für 30.000 gesunde Personen, die mit humanem Wachstumshormon aus Kadavern behandelt wurden, besteht eine erhöhte Gefahr, eine early-onset Amyloid- $\beta$-Pathologie zu entwickeln. Obwohl es bisher keine Beweise für eine Übertragbarkeit von Morbus Alzheimer gibt, sollten aufgrund der Befunde in dieser Studie iatrogene Übertragungswege von Prionen, wie etwa klinisches Besteck und Blutprodukte auch für Amyloid- $\beta$ und andere fehlgefaltete Proteine in Erwägung gezogen werden.

\section{- Kommentar von Erdem Tamgüney, Bonn}

\section{Lange Inkubationszeiten erschweren den Nachweis einer Übertragung}

Einzigartig sind Prionerkrankungen aufgrund der Tatsache, dass ihre Ätiologie nicht nur sporadisch oder familiär ist, sondern auch durch Infektion bedingt sein kann. Lange wurde spekuliert, ob andere Proteinopathien, wie zum Beispiel Morbus Alzheimer, ebenfalls eine infektiöse Komponente haben könnten [1]. Obwohl die Inokulierung von Hirnmaterial aus Alzheimer-Patienten in Mäuse, die das Amyloidprecursorprotein (APP) überexprimieren, Amyloid- $\beta$-Pathologie induziert, konnte eine Übertragbarkeit von Morbus Alzheimer unter Menschen bisher nicht gezeigt werden [2]. Auch gibt es Anzeichen, dass CJD-Prionen, wie sie in den Hormonpräparaten vorhanden waren, eventuell selbst eine Amyloid- $\beta$-Pathologie auslösen könnten, sodass weitere Studien notwendig sind, um die Beobachtungen von Jaunmuktane et al. zu verifizieren. Jedoch wird der Nachweis einer potenziellen Übertragung von Morbus Alzheimer durch die langen Inkubationszeiten von möglicherweise bis zu mehreren Jahrzehnten deutlich erschwert.
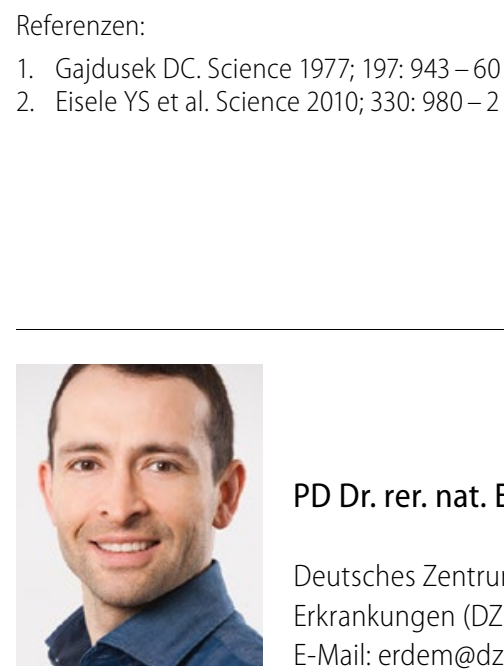

PD Dr. rer. nat. Erdem Tamgüney, Bonn

Deutsches Zentrum für Neurodegenerative Erkrankungen (DZNE)

E-Mail: erdem@dzne.de 